\title{
Highly Nonlinear Waves' Sensor Technology for Highway Infrastructures
}

\author{
Devvrath Khatri, ${ }^{1}$ Chiara Daraio, ${ }^{1,2}$ Piervincenzo Rizzo ${ }^{3}$ \\ ${ }^{1}$ Aeronautics (GALCIT) and ${ }^{2}$ Applied Physics, California Institute of Technology, 1200 E California \\ Boulevard, MC 105-50, Pasadena, CA 91125 USA, Tel: (626) 395-4479; Fax: (626) 449-6359 \\ ${ }^{3}$ Department of Civil \& Environmental Engineering, Univ. of Pittsburgh, 3700 O’Hara Street, \\ 949 Benedum Hall, Pittsburgh, PA, 15261, USA
}

\begin{abstract}
This paper describes preliminary results towards the development of an innovative NDE/SHM scheme for material characterization and defect detection based on the generation of highly nonlinear solitary waves (HNSWs). HNSWs are stress waves that can form and travel in highly nonlinear systems (i.e. granular, layered, fibrous or porous materials) with a finite spatial dimension independent on the wave amplitude. Compared to conventional linear waves, the generation of HNSWs does not rely on the use of electronic equipment (such as an arbitrary function generator) and on the response of piezoelectric crystals or other transduction mechanism. HNSWs possess unique tunable properties that provide a complete control over tailoring: 1) the choice of the wave's width (spatial size) for defects investigation, 2) the composition of the excited train of waves (i.e. number and separation of the waves used for testing), and 3) their amplitude and velocity. HNSWs are excited onto concrete samples and steel rebar. The first pilot study of this ongoing effort between Caltech and the University of Pittsburgh is presented.
\end{abstract}

Keywords: Highly nonlinear solitary waves, Nondestructive Evaluation, material characterization.

\section{INTRODUCTION}

The unavoidable aging of existing infrastructures demands that damage in new or aging systems can be detected at the earliest possible time in an effort to prevent life-threatening failures that can also lead to costly consequences for the taxpayers and the environment. Effective nondestructive evaluation (NDE) / structural health monitoring (SHM) techniques able to detect, locate and quantify structural damage, are therefore needed to ensure the performance, the proper response as well as the minimum maintenance costs of any engineering system.

The oil, gas and power generation industries make extensive use of pipes to process and transport their commodities. Corrosion and defective welds are prime sources of concern. In 2005, corroded pipes among the natural gas pipeline network, contributed to nearly $\$ 95$ million dollars damage ${ }^{1}$. In infrastructure applications, PS/PT structures offer dual advantages of exceptional life-cycle durability and (potentially) off-site prefabrication permitting rapid and minimally disruptive erection. Material degradation of the steel prestressing tendons, which are primary load-carrying components, is usually initiated by corrosion that results in a reduced load-carrying capacity of the structure and eventually collapse. Examples of such collapses were reported ${ }^{2-5}$. Accurately assessing the condition of existing pavements is essential for many transportation programs. In the U.S., most pavement structures were constructed decades ago, and much of the current investment in pavements is in maintenance and rehabilitation (M\&R) rather than in new construction. If the assessment is inaccurate, the estimated M\&R design may be either overly conservative or inadequate $^{6}$. From a sustainable standpoint, it is opportune to remark that whenever a whole or a part of an infrastructure is removed from service due to unacceptable level of damage or, in the worst case, due to a catastrophic collapse, the environment is three-times affected: 1) dismissed material has to be recycled (in the most optimistically scenario) or just merely discarded as landfill; 2) traffic congestion increases the pollutants emitted into the atmosphere; 3) any structural renewal or replacement implies the creation of a construction site that, although relatively short-lived in comparison to the structure's intended life, is a potential damaging exercise for the environment. Every year 140 million tons of construction and demolition waste are produced in the U.S. ${ }^{7}$.

Nondestructive Characterization for Composite Materials, Aerospace Engineering, Civil Infrastructure, and Homeland Security 2008, edited by Peter J. Shull, H. Felix Wu, Aaron A. Diaz, Dietmar W. Vogel, Proc. of SPIE Vol. 6934, 69340U, (2008) $\cdot 0277-786$ X/08/\$18 $\cdot$ doi: $10.1117 / 12.775848$ 
The above number remarks the need to develop and deploy inspection/monitoring methods that improve the strategies used today. Such progresses would benefit the industry, the state and federal agencies and, ultimately, the community. One of the most effective and widely accepted NDE/SHM methods is based on the propagation of elastic stress waves in the sonic or ultrasonic range. The propagation may depend on the wave frequency, material properties (e.g. composition and elastic modules), structural geometry (e.g. plate-like, rod-like, etc...), environmental conditions (temperature and humidity), or material stress (residual stress, applied stress).

This paper proposes a novel NDE/SHM paradigm based on the use of highly nonlinear solitary waves (HNSWs), ${ }^{8,9}$ able to detect defects at early stages and/or characterize the mechanical properties of a given structure. HNSWs are stress waves that can form and travel in highly nonlinear systems (i.e. granular, layered, fibrous or porous materials) with a finite spatial dimension that is independent on the wave amplitude. Compared to conventional linear waves, the generation of HNSWs does not rely on the use of electronic equipment (such as an arbitrary function generator) and on the response of piezoelectric crystals or other transduction mechanism. HNSWs offer a natural versatility (tunability) in terms of choice of wavelength, speed, amplitude, pulse shape and repetition rate. The choice can be achieved by a simple and reproducible experimental setup adaptable to specific application. The tunability of the solitary waves' properties can be exploited to develop, for the first time, an innovative NDE/SHM paradigm in which the HNSWs are used as the input waveforms. In addition, by relaxing the need for the power requirements (function generators) for ultrasonic actuation, the approach may facilitate the transition from tethered to wireless ultrasonic technology.

The NDE/SHM paradigm proposed here is schematized in Fig. 1. The highly nonlinear medium (represented in by a 1-D chain of beads) is used to generate a HNSW. The setup can be assembled with components at multiple length scales (i.e. from microparticles to macroscaled bearing balls) and in different geometries (i.e. alternating beads of different materials) to choose the desired wave propagation regime. The solitary wave then impinges on a bulk (Fig. 1a) or on a waveguide (Fig. 1b) structure. Both can be made by either linear, weakly nonlinear or highly nonlinear material. The type of waves being induced in the structures (bulk?, guided?, nonlinear?) with particular emphasis with the phenomena happening at the interface are currently under investigation in the framework of an ongoing effort between the Department of Civil and Environmental Engineering (CEE) at the University of Pittsburgh (Pitt) and the Departments of Aeronautics and Applied Physics at the California Institute of Technology (CalTech).

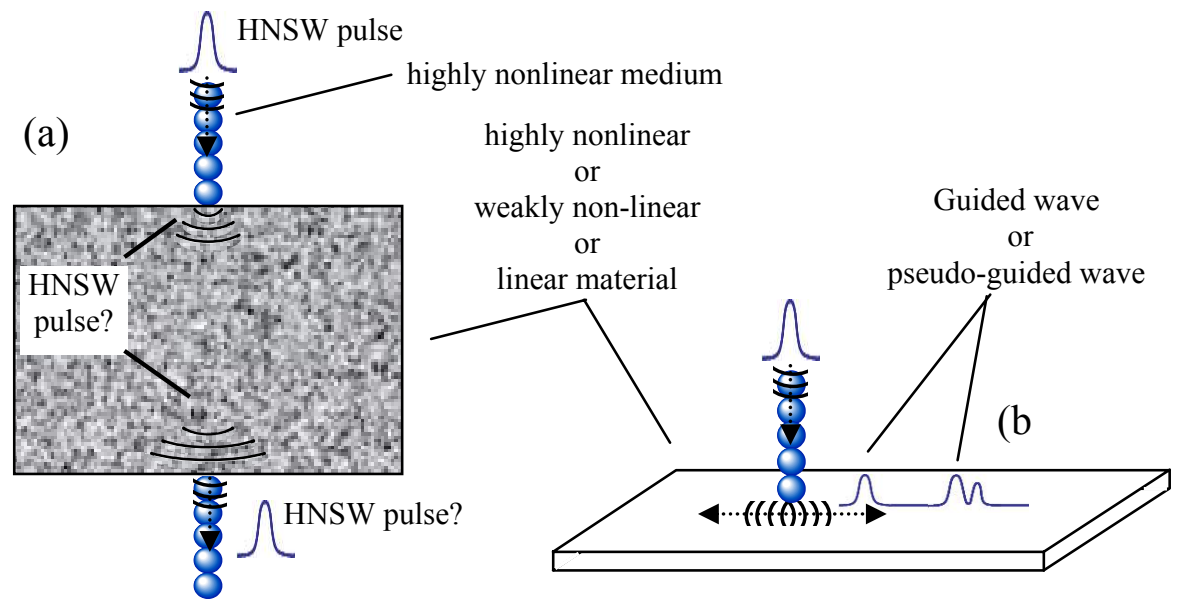

Figure 1. Propagation of HNSWs. (a) Bulk highly nonlinear, weakly nonlinear or linear medium. Wawe interaction at the interface at the generation and detection boundaries will be studied. (b) Generation of HNSWs in a waveguide.

\section{SOLITARY WAVES: BACKGROUND}

The NDE/SHM scheme proposed here is based on the use of (HNSWs). The existence of these waves and their propagation in solids was discovered while studying the shock absorption properties of granular matter. ${ }^{8}$ Since then, a new, general wave dynamic theory, supporting compact solitary waves that were never observed before, was derived for all uniform ${ }^{9}$ and heterogeneous ${ }^{10}$ materials that show a highly nonlinear force $(F)$ - displacement $(\delta)$. The general contact relation between the particles composing the system can be expressed as:

$$
F \cong A \delta^{n}
$$


where $A$ is a material's parameter and $n$ is the nonlinear exponent (with $n>1$ ), and $\delta$ is considered positive in compression. In tension $(\delta<0)$ the force becomes zero (particles can freely move apart from each other).

By "granular matter" we define an aggregate of particles in elastic contact with each other, preferably in linear or network shaped arrangements. One of the interesting features of the granular state is the negligible linear range of the interaction forces between neighboring particles resulting in an effective zero sound speed in the uncompressed material. This particular case makes the linear and weakly nonlinear continuum approaches based on Korteveg-de Vries (KdV) equation ${ }^{11}$ invalid and places granular materials in a special class according to their wave dynamics. When no static precompression is imposed on the granules, the resulting wave equation presents no linear part: this has led to the introduction of the concept of "sonic vacuum" (SV) - a medium where no characteristic sound speed is present $\left(c_{0}=0\right)$ and the traditional wave equation is no longer the basic equation for representing the wave dynamics. ${ }^{10}$ This highly nonlinear wave theory uncovers a huge potential for revolutionary applications in the field of dynamics and acoustic properties of materials. Highly nonlinear solitary waves have been experimentally and numerically observed in several works for the case of 1-D Hertzian granular materials. ${ }^{8-10,12-17}$

For granular materials the highly nonlinear behavior stems from the Hertz law for which the exponent $n$ in Eq. (1) is equal to 1.5. A similar response can be found in many other nonlinear systems and the theoretical formulation has been extended and generalized to all nonlinear exponents $n$, with $n>1 .{ }^{8-10}$ Examples of systems different from the Hertzian model are reported, for example, for irregular grains with conical contacts $(n=2)$, forests of vertically aligned carbon nanotubes $(n=2.2),{ }^{18}$ transverse vibration in a fiber with discrete particles $(n=3)$, plug chain gas-liquid systems $(n=3) .{ }^{9}$ Using the notation in ref. 9, the wave equation for the highly nonlinear system, derived from the Hertzian interaction law, is:

$$
u_{t t}=-c^{2}\left\{\left(-u_{x}\right)^{3 / 2}+\frac{a^{2}}{10}\left[\left(-u_{x}\right)^{1 / 4}\left(\left(-u_{x}\right)^{5 / 4}\right)_{x x}\right]\right\}_{x}
$$

where $u$ is the displacement, $a$ the particle's diameter, $c$ a material's constant, and the subscripts indicate the derivative. The constant $c$ in Eq. (2) is given by:

$$
c^{2}=\frac{2 E}{\pi \rho_{0}\left(1-v^{2}\right)}
$$

where $E$ is the Young's modulus, $\rho_{0}$ the density, and $v$ the Poisson coefficient. The generality of this highly nonlinear wave equation is given by the fact that it includes also the linear and weakly nonlinear wave equations. The classical KdV formulation used to describe weakly nonlinear systems can be extrapolated from Eq. (2) by adding static precompression to the system. Despite its apparent complexity the closed form solution of Eq. (2) can be obtained.

For the case of hertzian granular systems with no or very weak precompression acting on it, the exact solution exists in the form:

$$
\xi=\left(\frac{5 V_{p}^{2}}{4 c^{2}}\right)^{2} \cos ^{4}\left(\frac{\sqrt{10}}{5 a} x\right)
$$

where $\xi$ represents the strain and $\mathrm{V}_{\mathrm{p}}$ the system's velocity.

This solution demonstrates that in a highly nonlinear medium only two harmonics contribute to a stationary mode of propagation of the periodic signal. The solitary shape, if the initial prestrain $\xi_{0}$ is approaching 0 , can be taken as one hump of the periodic solution (Eq. 4) with finite wave length equal only to five particle diameters. In analogy with the $\mathrm{KdV}$ solitons, the highly nonlinear solitary waves are supersonic, which means that their phase velocity is larger than the initial sound velocity $\left(c_{0}\right)$ in the nonlinear medium (especially in the case of an uncompressed system, in which the $c_{0}=0$ ). A unique feature of the HNSWs is the independence of their width on the amplitude (their spatial size is always 5 particles diameter, no matter what wave amplitude or wave speed is present in the system). This property is quite different from the properties of weakly nonlinear KdV solitary waves ${ }^{8-10}$ and it is very important for the use of these solitary waves as information carriers and in signal transformation devices. as: ${ }^{17}$

The speed of the solitary wave $V_{\mathrm{s}}$ as nonlinear function of the maximum particle dynamic strain can be expressed 


$$
V_{s}=\frac{2}{\sqrt{5}} c \xi_{m}^{1 / 4}=0.6802\left(\frac{2 E}{a \rho^{3 / 2}\left(1-v^{2}\right)}\right)^{1 / 3} F_{m}^{1 / 6},
$$

where $F_{m}$ is the maximum dynamic contacts force between the particles in the discrete chain. This relationship uncovers a huge potential for revolutionary applications in the field of dynamics and acoustic properties of materials. One interesting characteristics of such waves predicted by the theory ${ }^{9}$ and validated numerically and experimentally ${ }^{16,17}$ is their tunability. By changing the mechanical and/or the geometrical properties of the high nonlinear medium supporting the formation of HNSWs, the shape and the properties of the traveling pulse can be tuned. In other words, the properties of the solitary waves in the highly nonlinear media can be "engineered" for a specific application. These "controllable" waves could then be used as new boundary conditions in the various structures for testing. The capability to generate a train of solitary waves rather than a single pulse is another promising aspect of the solitary wave's science.

The analytical expression for the tunability of the solitary waves speed derived from the discretization of the particles in the chain is expressed as follows: ${ }^{17}$

$$
V_{s}=0.9314\left(\frac{4 E^{2} F_{0}}{a^{2} \rho^{3}\left(1-v^{2}\right)^{2}}\right)^{1 / 6} \frac{1}{\left(f_{r}^{2 / 3}-1\right)}\left\{\frac{4}{15}\left[3+2 f_{r}^{5 / 3}-5 f_{r}^{2 / 3}\right]\right\}^{1 / 2} .
$$

where $F_{0}$ represents the static prestress (precompression) added to the system, $f_{\mathrm{r}}=F_{\mathrm{m}} / F_{0}$ and $F_{\mathrm{m}}$ is the maximum contacts force between the particles in the discrete chain.

The dependence of the solitary wave properties on the materials parameters is shown in Eq. (5) for a non-prestressed system and in Eq. (6) for a prestressed system. Another interesting feature of the HNSWs is determined by the fact that the system is size independent and the solitary waves can therefore be scalable to any dimension, according to the needs of each specific application.

According to Eqs. (5) and (6), the tunability of the HNSW can be achieved varying one or more parameters of the nonlinear medium. For instance, as shown later, by increasing the particle size of the highly nonlinear medium the wavelength increases, and the wave speed and amplitude decrease. Also, by adding periodic heterogeneities the wave properties have been demonstrated to vary, and the wavelength increase accordingly. ${ }^{19,10}$

The tunability can be exploited to replace some of the electronic equipment, such as function generators, necessary to excite stress wave of given shape and wavelength. This aspect would relax some of the power demands in ultrasonic actuation and ease the transition from tethered to wireless technology. In addition, the high-sensitivity of wave amplitude and wave speed to the state of stress state can be exploited to dramatically improve the estimation of applied stress obtained by conventional acoustoelastic methods. Rizzo and co-authors demonstrated ${ }^{20,21}$ that the variation of the group velocity of a longitudinal guided wave propagating in a seven-wire steel strand varies less than one percent over a stress range of $0-1.25 \mathrm{GPa}$.

The use of solitary waves for defect and impurity detection in granular media was proposed and tested ${ }^{13,22}$, showing excellent promises for applicability. Other studies demonstrated the sensitivity of solitary waves to materials properties such as elastic modules ${ }^{12,14}$ and applied stress ${ }^{17}$.

Hong and $\mathrm{Xu}^{13}$ demonstrated a dependency of the velocity and shape of the backscattered signal on the presence of light and heavy impurities in a granular chain. Variation was observed in the region of light impurity, while no variation was observed for the case of a heavy impurity. This difference may be used to discriminate nondestructively between light and heavy impurities in granular media. Coste and co-authors ${ }^{12}$ and Daraio and co-authors ${ }^{14,15}$ studied numerically and experimentally the propagation of HNSW in a one-dimensional highly nonlinear system assembled from chains of stainless-steel, glass, brass, nylon, polytetrafluoroethylene (PTFE) and Parylene coated steel beads. As predicted by the theoretical formulation, the numerical and experimental validation showed a significant difference in the speed and amplitude of the supported solitary waves as a function of the materials parameters. In addition, Daraio and co-authors experimentally demonstrated ${ }^{16}$ that a pulse traveling in a system composed of alternating short chains of hard and soft beads (that can be interpreted as defects) induce dramatic changes in the properties of the traveling pulse.

\section{EXPERIMENTAL RESULTS}

To excite HNSWs a chain of low-carbon steel beads (diameter equal to $4.76 \mathrm{~mm}$ ), confined into a plastic guide, was assembled from a number of balls $\left(N_{\mathrm{b}}\right)$ ranging from 10 to 35 . The highly nonlinear waves formed and propagated through the system and the pulses were induced into a steel $1018 \mathrm{rod}$. The rod was $490 \mathrm{~mm}$ long and $18 \mathrm{~mm}$ in diameter. 
The chain was vertically mounted on top of one bar's cross-section and a Physical Acoustic PICO sensor (100-800 kHz resonant frequency) was attached at the opposite cross-section by using PCB Petro Vax. The setup was designed to excite and detect longitudinal sonic and ultrasonic guided waves. The dispersion curves associated with the group velocity of a $18 \mathrm{~mm}$ diameter steel rod in vacuum are plotted in Fig. 2. The following material properties are assumed for steel $1018 \mathrm{c}_{\mathrm{L}}=5.890 \mathrm{~mm} / \mu \mathrm{sec}, \mathrm{c}_{\mathrm{T}}=3.233 \mathrm{~mm} / \mu \mathrm{sec}$ and $\rho=7843 \mathrm{~kg} / \mathrm{m}^{3}$. The first two longitudinal and flexural modes are shown in Fig. $2 .^{23}$

The stress waves (first arrival and the two echoes) detected by the bottom transducers are illustrated in Fig. 3a for a number of particles $\left(N_{\mathrm{b}}\right)$ in the chain equal to $10,15,20,30$, and 35 . As $N_{\mathrm{b}}$ increases the wave amplitude decreases due to the effects of dissipation in the systems. Although the signal amplitude decreases with the increase of $N_{\mathrm{b}}$ due to the reduced amount of kinetic energy that is transferred from the striker to the chain, it is evident that shapes of propagating pulses are identical. This validates the repeatability of the HNSWs-induced pulse. In addition by that changing the chain configuration (number of beads) the amplitude can be tuned, without affecting the shape, frequency or time arrival of the

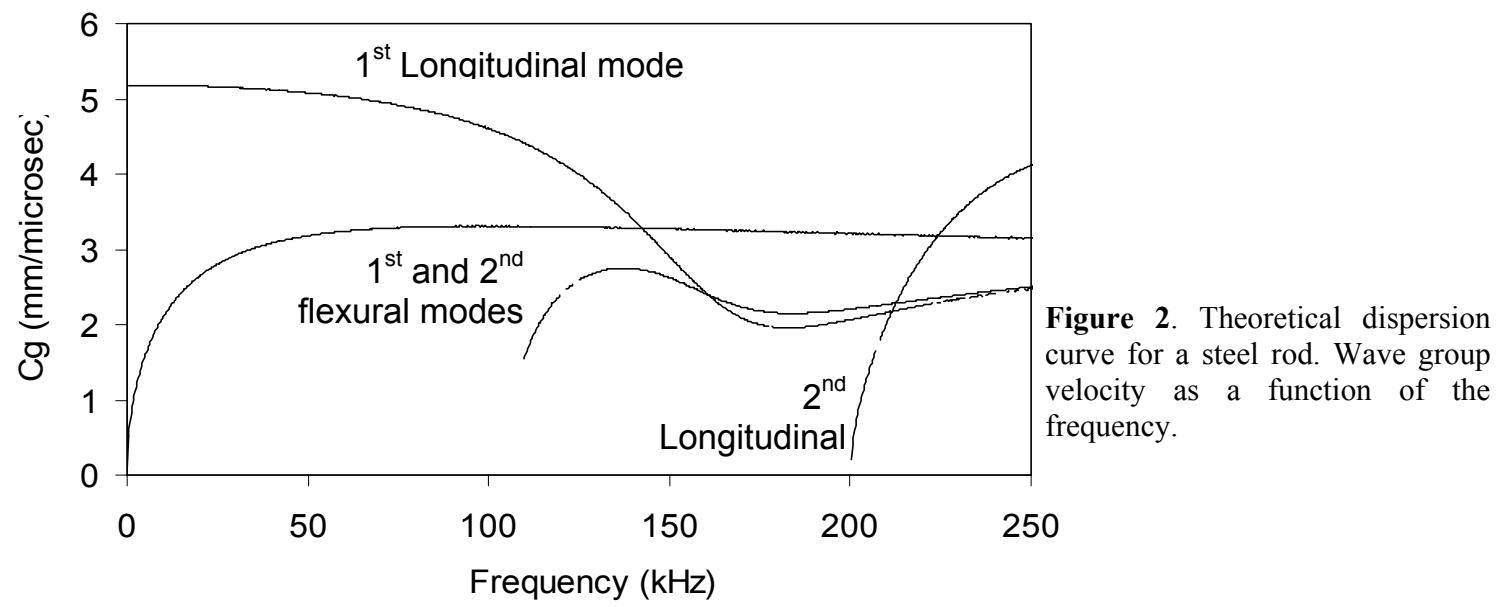

induced wave. The guided wave velocity was found equal to $5.175 \mathrm{~km} / \mathrm{sec}$ (at $25 \mathrm{kHz}$ ) which match well with the theoretical value predicted in the dispersion curve. ${ }^{23}$ This suggests that the HNSWs-induced pulse is converted into a linear longitudinal guided wave as it travels along the rod.

A PCB miniature hammer was used to excite the stress waves in a similar configuration. The time waveforms from two separate excitations are illustrated below. It can be seen that the shape of the signals is similar but not identical. This is associated with the variability of the impact from the hammer. Furthermore the amplitude of such amplitude changes and this change is not related to the setup (as for the bead chain) but it is associated to the operator skill to provide the same impact duration. By comparing Fig. $3 \mathrm{~b}$ to Fig. $3 \mathrm{a}$ it can be noted that the stress waves induced are qualitatively similar.

An additional set of preliminary results were obtained using a chain of 20 stainless steel particles as impulse exciter
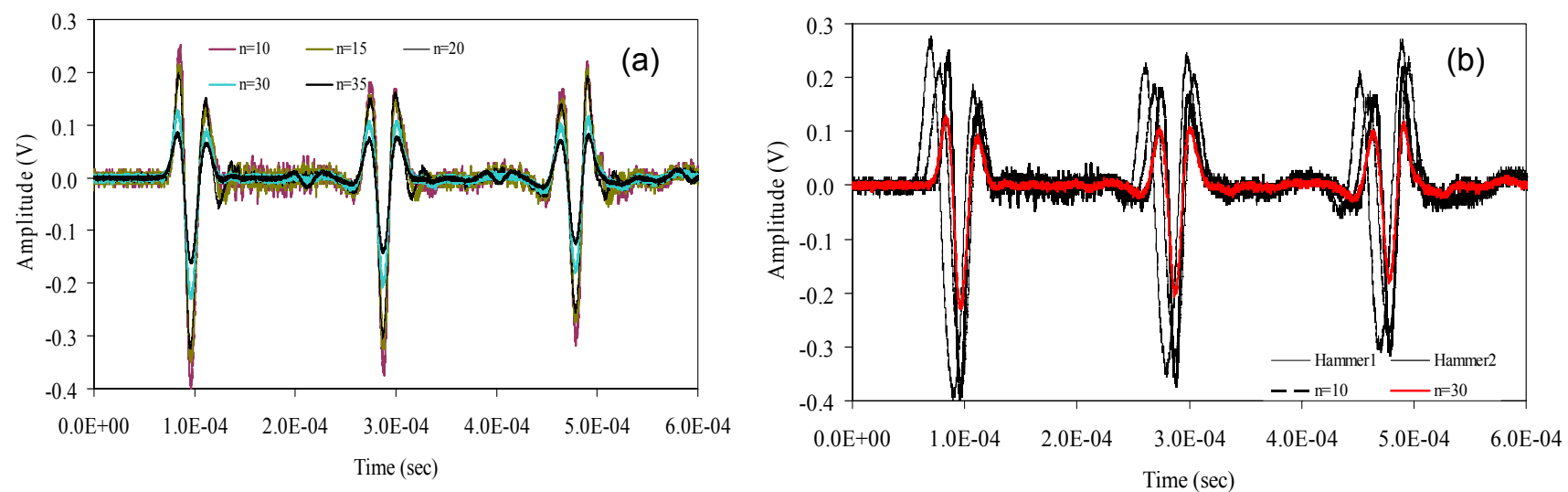

Figure 3. HNSWs-induced pulses into a steel rod. (a) Pulses generated with a variable number $\left(N_{\mathrm{b}}\right)$ of beads into a chain of stainless steel beads. (b) Pulses generated by impacting a miniature hammer and by using $N_{\mathrm{b}}=10,30$ from (a). 
positioned on top of $4.76 \mathrm{~mm}$ steel rods. The chains used for the study consisted of uniform beads with different diameters assembled inside a four-rod stand (a guide) as described in details in Ref [10]. We selected stainless steel bead (316 steel, McMaster-Carr catalog) with diameter $\mathrm{a}=4.76 \mathrm{~mm}$ and mass $0.45 \mathrm{~g}$ and smaller stainless steel bead (316 steel, McMaster-Carr catalog) with diameter $\mathrm{a}=2.38 \mathrm{~mm}$. We excited HNSWs in such chains impacting its free end with one $4.76 \mathrm{~mm}$ stainless steel bead (used as a striker) and monitored the propagation of such waves within the chain, at the interface between the chain and the rod, and at the bottom of the entire set up using piezo-gauges. Calibrated piezosensors ( $\mathrm{RC} \sim 10^{3} \mu \mathrm{s}$ ) were connected to a Tektronix oscilloscope to detect force-time curves. Piezo-gauges made from lead zirconate titanate (square plates with thickness $0.5 \mathrm{~mm}$ and $3 \mathrm{~mm}$ side) with nickel-plated electrodes and custom micro-miniature wiring (supplied by Piezo Systems, Inc.) were embedded inside steel particles in the chains or sandwiched between $4 \mathrm{~mm}$ diameter, $2 \mathrm{~mm}$ thick steel disks and placed at the rod's extremities.

To compare the effects of the HNSWs traveling in the system and their ability to detect potential defects in the steel rod, we machined a $2 \mathrm{~mm}$ deep notch in the middle section of one of the steel rods used for the measurements. A schematic diagram of the experimental set up is shown in Fig. 4. We compare the input waveforms generated in the two set ups, and the one transmitted across the damaged and undamaged steel rods. The results obtained for the set up described in Fig. 4(a) are shown in Fig. 5(a) for the pristine rod and in Fig. 5(b) for the damaged rod. It is clear that the pulse excited is very repeatable and the presence of the defect significantly alters the transmitted wave's amplitude and shape. Similarly, Fig. 5(c,d) show the results obtained from the set up depicted in Fig. 4(b) in a pristine and damaged rod respectively. The reduction of the particles' diameter implies a dramatic change of the input signal under identical initial excitation (compare for example, the curves corresponding to the signal recorded from the interface sensor in Fig. 5(a) and 5(c)). Such changes are reflected on the frequency response of the system, which increases proportionally with the reduction of the bead's diameter. This suggests that the proposed method for NDE/SHM can be scaled according to the potential type of defects and stress

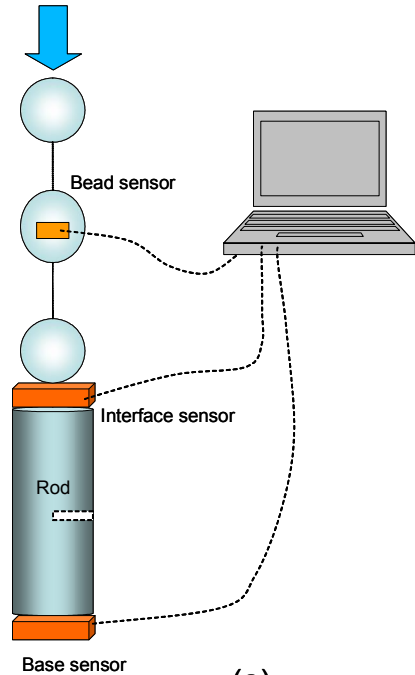

(a)

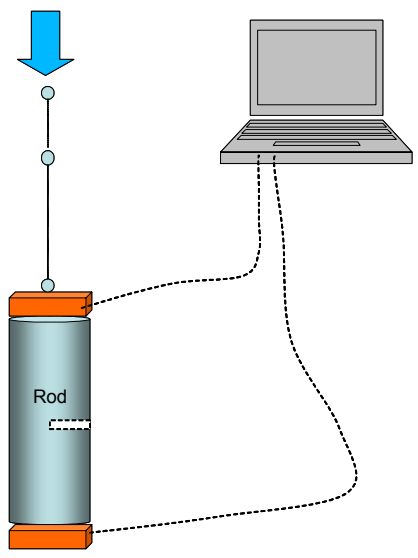

(b)

Figure 4. Experimental set up consisting of 20 vertically aligned stainless steel particles, positioned on top of a $4.76 \mathrm{~mm}$ diameter steel rod. The rectangular elements (in orange) indicate the position of the piezogauges used for monitoring the signal propagation in the system. (a) System with particle diameter of $4.76 \mathrm{~mm}$ and (b) $2.38 \mathrm{~mm}$. The arrows show the direction of impulse excitation, obtained using a $4.76 \mathrm{~mm}$ stainless steel bead as a striker dropped from $\sim 3 \mathrm{~cm}$ height.

Strongly nonlinear materials allow a significant change of speed of signal propagation and pulse shape by adding a static compressive force. ${ }^{9,17}$ In our experiments the tunability of the signal propagating through the chains was achieved by adding a magnetically induced preload. A neodymium-iron-boron ring magnet (internal and external diameters 11.43 $\mathrm{mm}, 53.34 \mathrm{~mm}$, respectively, with mass $242.2 \mathrm{~g}$ from Dexter Magnetics Technologies) was placed around the setup containing the chain and held in place by the magnetic interaction with a magnetic steel particle (diameter $a=5.0 \mathrm{~mm}$ and mass $m_{\mathrm{m}}=0.51 \mathrm{~g}$ ), placed on the top of the chain.

Throughout the whole chain the static compressive force induced by the magnet was $2.38 \mathrm{~N}$, in additional to nonuniform gravitational preload created by the beads. We tested the effect of static precompression on the excitation of the HNSWs in the chain and on the transmission of the impulse inside the steel rod. The experimental set up and the corresponding preliminary results obtained in this case are shown in Fig. 6 (a-c). It is evident that the presence of the magnetic preload changes significantly the pulse speed within the chain (increasing the wave speed by $\sim 20 \%$ with the considered impact conditions). It also generates the presence of secondary oscillations (see for example the curve corresponding to the bead sensor in Fig. $6(\mathrm{~b}, \mathrm{c}))$. The combination of these effects causes the transmitted signal to be also modified, as it is evident comparing the curves of Fig. 5(a,b) for the non-precompressed case, with the one in Fig. $6(b, c)$ for the precompressed one. The capability to detect defects with the generated HNSWs can be verified comparing the base sensors curves in Fig. 5(a and b) and Fig. 5(c and d) for the uncompressed large and small beads systems respectively. 

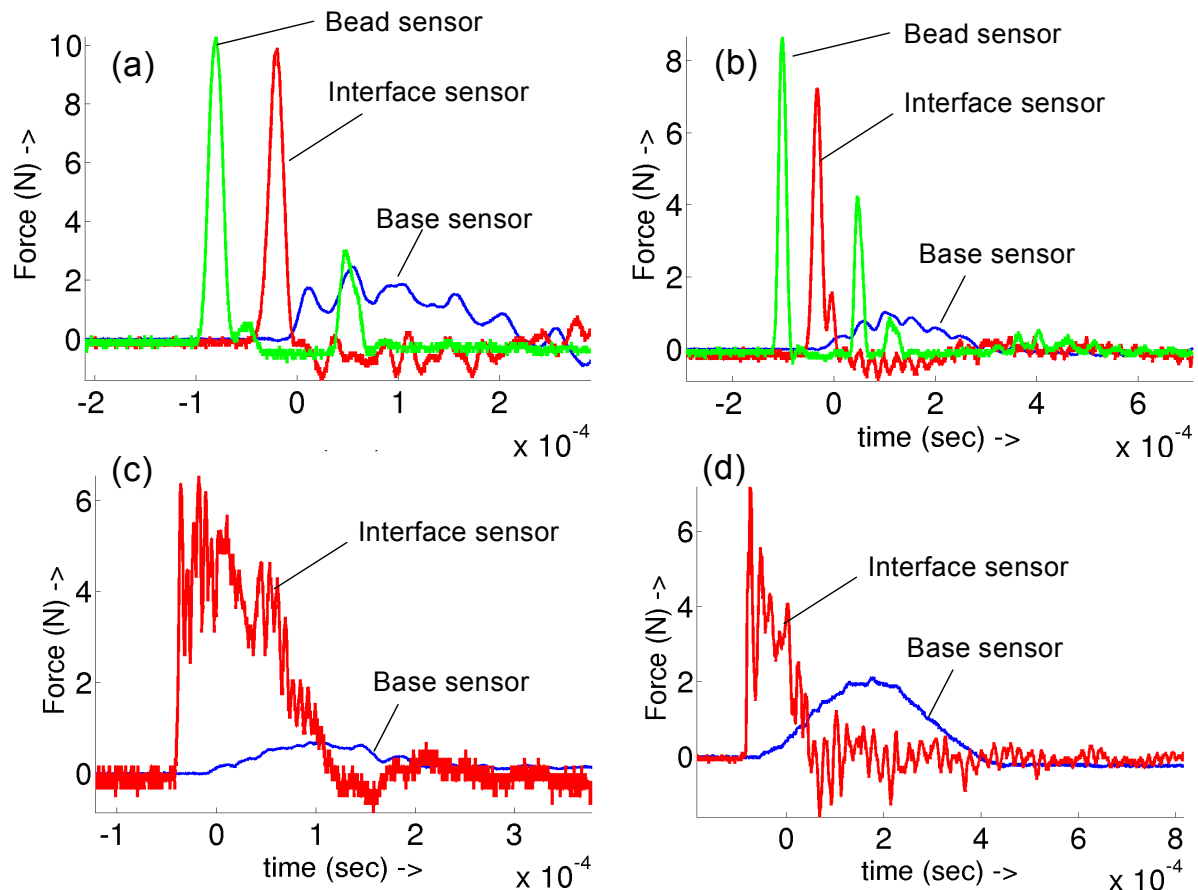

Figure 5. Experimental curves obtained for the set ups described in Fig. 4, corresponding to (a) 4.76 mm diameter beads on a pristine steel rod, (b) $4.76 \mathrm{~mm}$ diameter beads on a damaged steel rod, (c) $2.38 \mathrm{~mm}$ diameter beads on an undamaged steel rod and (d) $2.38 \mathrm{~mm}$ diameter beads on a damaged rod. In (a) and (b) sensors are positioned in one of the central beads composing the chain (curve labeled "bead sensor"), at the interface and at the base below the steel rod. In (c) and (d) sensors are positioned only at the interface and at the rod's base. The impulses were generated dropping a $0.45 \mathrm{~g}$ steel bead from a height of $\sim 3 \mathrm{~cm}$ on the top particle of the chain.

Similarly successful defect detection is evident also in the precompressed system case: compare the base sensor curves of Fig. $6(\mathrm{~b}$ and $\mathrm{c}$ ) for the pristine and notched rod respectively.

\section{CONCLUSIONS}

This paper proposes a novel NDE/SHM paradigm based on the use of highly nonlinear solitary waves (HNSWs), able to detect defects at an early stage and/or characterize the mechanical properties of a given structure. HNSWs are stress waves that can form and travel in highly nonlinear systems (i.e. granular, layered, fibrous or porous materials) with a finite spatial dimension that is independent on the wave amplitude. Compared to conventional linear waves, the generation of HNSWs does not rely on the use of electronic equipment (such as an arbitrary function generator) and on the response of piezoelectric crystals or other transduction mechanism. The first pilot study of this ongoing effort between Caltech and the University of Pittsburgh is presented. A chain of beads is used to induce stress waves into a steel rod. The test have demonstrated the repeatability of the newly adopted setup and has demonstrated an excellent agreement between the pulses excited with miniature hammer and pulses excited with the chain of beads, with
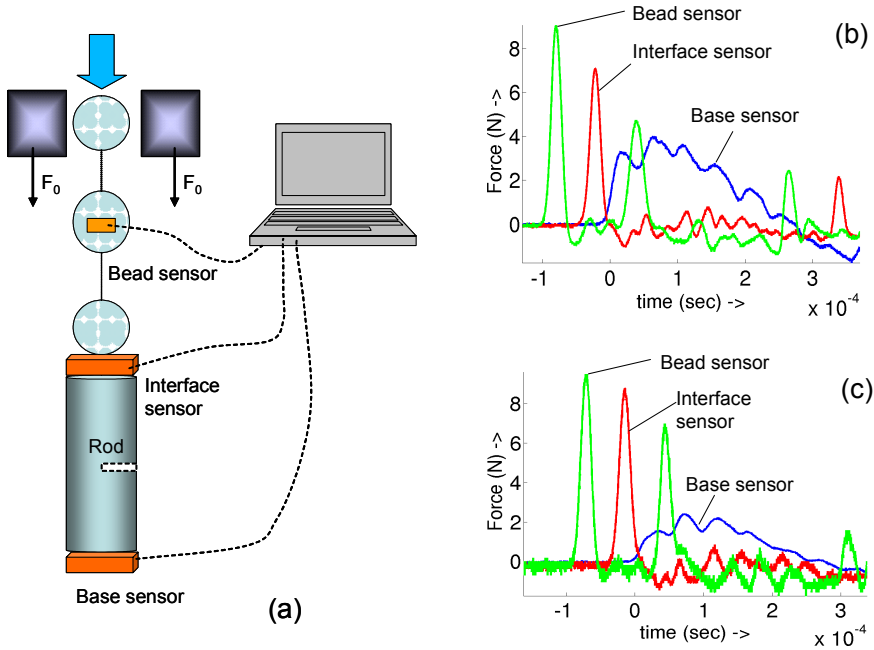

Figure 6. (a) Experimental set up consisting of 20 vertically aligned stainless steel particles, positioned on top of a $4.76 \mathrm{~mm}$ diameter steel rod with added static precompression $\left(F_{0}=2.38 \mathrm{~N}\right)$. The rectangular elements (in orange) indicate the position of the piezogauges used for monitoring the signal propagation in the system. The arrow shows the direction of impulse excitation, obtained using a $4.76 \mathrm{~mm}$ stainless steel bead as a striker dropped from $\sim 3 \mathrm{~cm}$ height. (b) Force-Time curves corresponding to the sensors indicated in the set up comprising a pristine steel rod and (c) a damaged rod. 
superior tunability and reproducibility in the latter. Tunability over the pulse excitation and transmission was demonstrated varying the beads diameter and adding static precompression.

\section{ACKNOWLEDGMENTS}

Chiara Daraio and Piervincenzo Rizzo acknowledge the support of Caltech and Pitt startup funds, respectively.

\section{REFERENCES}

[1] Office of Pipeline Safety. Pipeline Statistics - Natural Gas Accidents for Transmission Operators Yearly Summary for 2005. http://ops.dot.gov/stats/tran_sum.htm

[2] Chase, S.B., "Smarter Bridges, Why and How?", Smart Materials Bulletin, 2, 9-13, 2001

[3] Parker, D., "Pacific Bridge Collapse Throws up Doubt on Repair Method", New Civil Engineer, 12/12/96 and 12/26/96, 3-4, 1996.

[4] Woodward R.J., "Collapse of Ynys-y-Gwas Bridge, West Glamorgan”, Proc - Institution of Civil Engineers I, 84, 635-669, 1988.

[5] Harries, K., Earls, C., Gostautas, R., and Stull, C, "Full-scale testing program on de-commissioned girders from the lake view drive bridge," PITT Report CE/ST-33, FHWA-PA-2006-008-EMG001, PennDOT (2006).

[6] Grotea, K., Hubbard, S., Harvey, J., and Rubina, Y., "Evaluation of infiltration in layered pavements using surface GPR reflection techniques," Journal of Applied Geophysics, 57, 129-153, 2005.

[7] Kibert, C., Sustainable Construction, Green Building Design and Delivery, John Wiley \& Sons, Inc., Hoboken, N.J., 2005.

[8] Nesterenko, V.F., Prikl. Mekh. Tekh. Fiz. 5, 136, 1983. In Russian. (J. Appl. Mech. Tech. Phys. 5, 733, 1984).

[9] Nesterenko, V.F., Dynamics of Heterogeneous Materials, Ch.1, Springer-Verlag, NY, 1983.

[10] Porter, M.A.; Daraio, C.; Herbold, E.B.; Szelengowicz, I.; Kevrekidis, P.G. "Highly nonlinear solitary waves in phononic crystal dimers" Physical Review E, 77, 015601(R), 2008.

[11] Korteweg, D.J., and de Vries, G., "On the change of form of long waves advancing in a rectangular canal, and on a New type of long stationary Waves," London, Edinburgh and Dublin Philosophical Magazine and Journal of Science, ser. 5, 39, 422-443, 1895.

[12] Coste, C., Falcon, E., and Fauve, S., "Solitary waves in a chain of beads under Hertz contact," Phys. Rev. E, 56, 6104-6117, 1997.

[13] Hong, J. and Xu, A, "Nondestructive identification of impurities in granular medium," Appl. Phys. Lett., 81, 48684870, 2002.

[14] Daraio, C., and Nesterenko, V.F., Herbold, E.B., and Jin, S. "Strongly nonlinear waves in a chain of Teflon beads," Phys. Rev E, 72, 016603, 2005.

[15] Daraio, C., and Nesterenko, V.F. (2006). "Strongly nonlinear waves in a chain of polymer coated steel beads," Phys. Rev. E; 73, 026612.

[16] Daraio, C., and Nesterenko, V.F.; Herbold, E., and Jin, S., "Energy Trapping and Shock Disintegration in a Composite Granular Medium,” Phys. Rev. Lett., 96, 058002, 2006.

[17] Daraio, C., and Nesterenko, V.F.; Herbold, E. and Jin, S, "Tunability of solitary wave properties in one dimensional strongly nonlinear phononic crystals," Phys. Rev. E, 73, 026610, 2006

[18] Daraio, C., and Nesterenko, V.F.; Jin, S.; Wang, W. and Rao, A.M., "Impact Response by a Forest of Coiled Carbon Nanotubes," Journal of Applied Physics, 100, 064309, 2006.

[19] Porter, M.A.; Daraio, C.; Szelengowicz, I.; Herbold, E.B., and Kevrekidis, P.G. (2007). "Highly Nonlinear Solitary Waves in Heterogeneous Periodic Granular Media," Physica D, (submitted).

[20] Lanza di Scalea, F., Rizzo, P., and Seible F., "Stress Measurement and Defect Detection in Steel Strands by Guided Stress Waves", ASCE Journal of Materials in Civil Engineering, Special Issue on NDE, 15 (3), 211-304, 2003.

[21] Rizzo, P., and Lanza di Scalea, F. (2004). "Monitoring in Cable Stays via Guided Wave Magnetostrictive Ultrasonics," Materials Evaluation, 62(10), 1057-1065.

[22] Sen, S., Manciu, M., and Wright J.D. (1998). "Soliton like pulses in perturbed and driven Hertzian chains and their possible applications in detecting buried impurities," Phys. Rev. E, 57, 2, 2386-2397.

[23] Rizzo, P., Health Monitoring of Tendons and Stay Cables for Civil Structures, PhD Dissertation, 2004. 